\title{
Optimization of the final settings for the Space-borne Hard X-ray Compton Polarimeter POLAR
}

\author{
Hualin Xiao*, Wojtek Hajdas, Radoslaw Marcinkowski on behalf of POLAR \\ collaboration \\ Paul Scherrer Institute \\ E-mail: hualin.xiao@psi.ch
}

POLAR is a compact wide field space-borne detector dedicated for precise measurements of the linear polarization of hard X-rays emitted by transient sources in the energy range from $50 \mathrm{keV}$ to $500 \mathrm{keV}$. It consists of 1600 plastic scintillator bars grouped in 25 detector modules that are used as gamma-ray detection material. Its energy range sensitivity is optimized for detection of the prompt emission photons from the gamma-ray bursts. Measurements of the GRB polarization provide unique information on emission mechanisms as well as on composition and structure of the GRB jets. The POLAR instrument was developed by international collaboration of Switzerland, China and Poland. It was launched in space on-board the China Space Laboratory TG-2 on September 15th, 2016. Based on the ground calibration data, several high voltage and threshold settings were calculated and verified in order to obtain various energy ranges and optimized signal to background conditions for different measurement purposes. In this paper we present optimization procedure details and current test results.

35th International Cosmic Ray Conference - ICRC2017

10-20 July, 2017

Bexco, Busan, Korea

${ }^{*}$ Speaker. 


\section{Introduction}
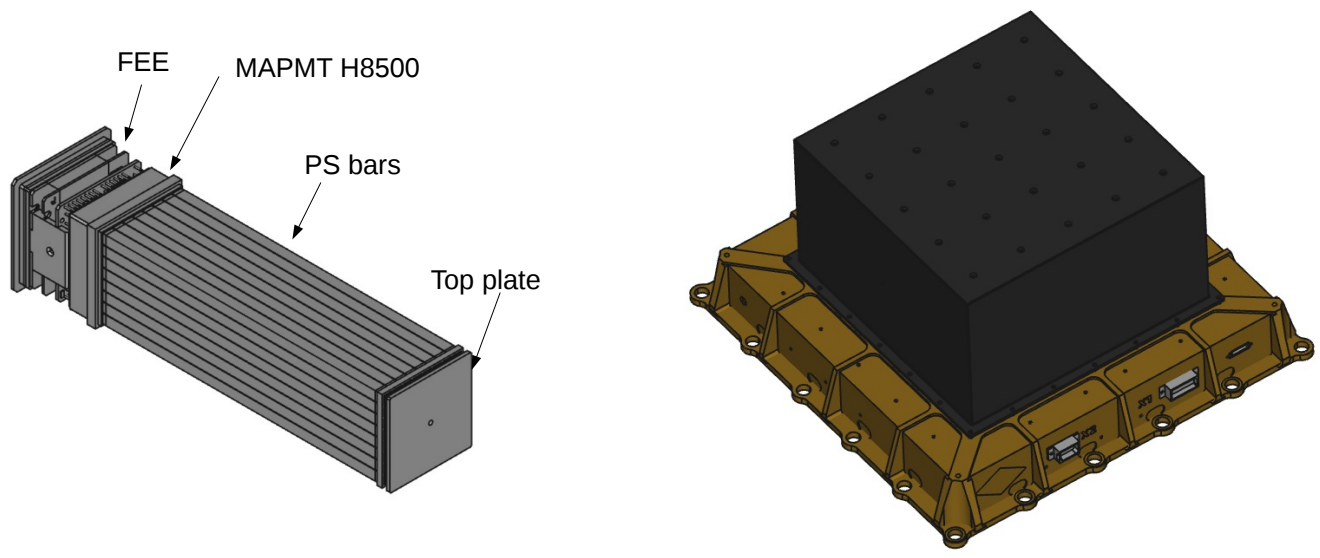

Fig. 1: POLAR detector module structure (left) and POLAR flight model (right). Each module has 64 PS bars $\left(5.8 \times 5.8 \times 176 \mathrm{~mm}^{3} \mathrm{each}\right)$ readout by a 64 channel MAPMT (Hamamatsu H8500) and its front-end electronics. Note that the $1 \mathrm{~mm}$ thick carbon fibre socket is not shown in the left panel. The full instrument consists of 25 identical modules.

GRBs are sudden flashes of gamma-rays which appear randomly in the sky and for a few seconds outshine all other gamma-ray sources. They are produced at cosmological distances and are considered as the brightest events in the universe after the Big Bang. In the past 40 years many instruments have performed extensive studies of GRBs but both their creation mechanisms and their progenitors are still uncertain. Direct measurements of the linear polarization in the gammaray band are thought to have a great potential to distinguish between different theoretical models by providing missing information needed to pin down emission mechanisms as well as composition and geometry structure of the GRB jets [1,2].

POLAR is a compact wide-field space-borne Compton polarimeter dedicated for precise measurements of the linear polarization of hard $\mathrm{X}$-rays emitted by transient sources in the energy range from $50 \mathrm{keV}$ to $500 \mathrm{keV}$. POLAR has both, a large effective detection area $\left(\sim 80 \mathrm{~cm}^{2}\right)$ and a wide field of view ( $\sim 1 / 3$ of full sky). For stronger GRB events its minimal detectable polarization (MDP) may be better than 10\%. Due to its wide field of view, the instrument is also capable of polarization measurements in solar flares. POLAR instrument was built by a collaboration of institutes from Switzerland, China and Poland. It was launched into space on September 15th, 2016 on-board the Chinese Space Laboratory TG-2 with a goal to reach up to 3 years long observation period.

POLAR detector consists of 1600 plastic scintillator bars grouped in 25 identical detector mod- 
ules. Each module consists of $8 \times 8$ PS bars, a 64 pixel multi-anode PMT (Hamamatsu MAPMT H8500) and a readout front-end electronics as shown in the left panel of Fig. 1. Each bar has a dimension of $5.8 \times 5.8 \times 176 \mathrm{~mm}^{3}$ and is wrapped with a high reflective foil (ESR). All 64 bars are coupled to the MAPMT via an optical pad. The FEE consists of three stacked Printed Circuit Boards (PCBs): high voltage divider board, signal processing board and power supply and interfacing board. The high voltage divider consists of twelve $470 \mathrm{k} \Omega$ resistors distributing high voltages to the MAPMT dynodes. The signal processing board consists of a ASCI chip (IDEAS VA64) with 64 separate readout channels, ADC, DAC, FPGA, and a temperature sensor. The third board has a low voltage supply circuit and power and signal connecting interface. All 64 channels share the same high voltage divider; while each module has an individual high voltage supply. The discriminators in the ASIC have a common threshold voltage value apart of small range trimmers. Each detector module is packed with a $1 \mathrm{~mm}$ thick carbon fibre socket. All 25 modules are managed by the Central Task Processing Unit (CT). In addition, CT also manages power supplies, makes decisions on triggers and handles internal and external communication. All above subsystems together with power supplies and interfacing parts are enclosed in a Carbon box. It is shown in the right panel of Fig. 1. The whole instrument is mounted on the outside panel of the space-lab.

The main goal of POLAR is realized by measuring the anisotropy of the azimuthal Compton scattering as described in the Klein-Nishina equation:

$$
\frac{\mathrm{d} \sigma}{d \Omega}=\frac{r_{\mathrm{e}}^{2}}{2}\left(\frac{E^{\prime}}{E}\right)^{2}\left(\frac{E^{\prime}}{E}+\frac{E}{E^{\prime}}-2 \sin \theta^{2} \cos \eta^{2}\right),
$$

where $r_{\mathrm{e}}$ is the classical radius of the electron, $E$ and $E^{\prime}$ are the energy of the incident photon and the scattered photon, respectively, $\theta$ is the scattering angle between initial and final photon direction, and $\eta$ is the azimuthal scattering angle between the initial polarization vector and the direction of the scattered photon. The distribution of $\eta$, also called modulation curve, can be parametrized as:

$$
f(\eta)=k \cdot\{1+\mu \cos (2(\eta-\theta)+\pi))\}
$$

where $\theta$ is the polarization angle, $k$ is the normalization factor and $\mu$ is the modulation factor which is proportional to the polarization degree. The polarization degree can be acquired from $p=\mu / \mu_{100}$, where $\mu_{100}$ is the modulation factor for $100 \%$ polarized beam. Detailed descriptions of the POLAR principle and the polarization reconstruction methods can be found in Refs. [3, 4, 5].

In-flight energy calibration is carried out with four weak ${ }^{22} \mathrm{Na}$ positron sources placed inside of POLAR. As the sources emit two back-to-back annihilation photons, the selection of geometrically aligned coincidence events allows for proper calibration of each channel in the instrument. Energy calibration with other high voltage settings can be obtained using scaled energy conversion factors and experimentally determined high voltage dependence. The annihilation gamma-ray pairs from calibration sources produce much higher energy depositions in scintillator bars than most of gamma-rays from GRBs. Therefore, several high voltage and threshold settings are needed for comprehensive in-flight energy calibration and proper GRB observations. Before launching of POLAR, the high voltage vs. gain relation as well as threshold values were calibrated and several optimized settings for various measurement types were provided. This paper presents details of POLAR calibrations and describes optimization methods for its final settings. 


\section{Calibration of POLAR flight model}

\subsection{Energy calibration}

MAPMTs of POLAR operate using an equally distributed voltage divider. Their gain factor in relation to applied high voltage $V$ is given by the following equation [6]:

$$
G=a^{n}\left(\frac{V}{n+1}\right)^{k n},
$$

where $a$ is a constant, $n$ is the number of dynode stages and $k$ is a constant determined by the structure and material of the PMT. The typical value of $k$ is 0.7 [6]. In the case of POLAR, $n$ is equal to 12. According to Eq. (2.1), the gain $G$ is proportional to the kn-th power of the high voltage. It is reasonable to assume that the energy response of POLAR detector close to its typical operating condition is linear. The energy conversion $c$ (in units of ADC channel / keV) can be given by

$$
c(V)=\frac{E_{\text {meas }}}{E_{\mathrm{vis}}}=b G=\alpha\left(\frac{V}{n+1}\right)^{k n},
$$

where $b$ is a constant, $V$ is the high voltage, $\alpha$ is equal to $b a^{n}, E_{\text {vis }}$ and $E_{\text {meas }}$ are the energy deposition (in units of $\mathrm{keV}$ ) and the recorded energy deposition (in units of ADC channel) respectively. In order to determine the dependence between energy conversion factors and high voltage values, POLAR flight model (FM) was tested with the ${ }^{137} \mathrm{Cs}$ source at six high voltage values from $610 \mathrm{~V}$ to $660 \mathrm{~V}$ with a step of $10 \mathrm{~V}$. Fig. 2 shows calculated energy conversion factors of all 1600 channels using Eq. (2.2) parameterized with the calibration data. In this case the high voltage values of all modules were equal to $700 \mathrm{~V}$. More details about the method used in this study and the data analysis are available in Refs. [7] and [8].

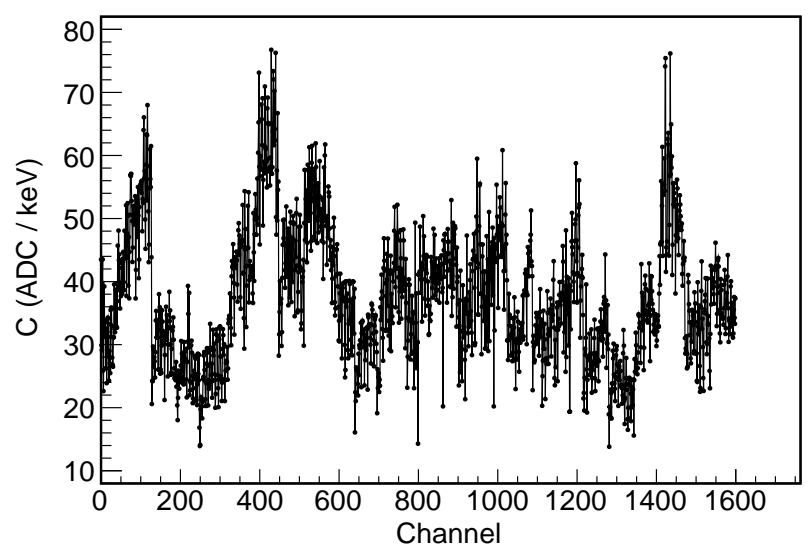

Fig. 2: Calculated energy conversion factors of all 1600 channels using Eq. (2.2) parameterized in laboratory calibrations with ${ }^{137} \mathrm{Cs}$ gamma-ray source. High voltage values of all modules were set to $700 \mathrm{~V}$.

\subsection{Threshold calibration}

Precise knowledge of thresholds in all 1600 channels of POLAR is crucial as the value of its modulation factor computed for GRB polarization reconstruction is threshold dependent. The 
ASICs in POLAR front-end electronics have a discriminator for each module with 64 channels. The discriminator can be set to the appropriate voltage value $\left(V_{\mathrm{thr}}\right)$ of the threshold by sending commands to its FEE. The real threshold value $E_{\text {thr }}$ in units of $\mathrm{keV}$ can be given by

$$
E_{\mathrm{th}}=c \cdot\left(p_{0}+p_{1} V_{\mathrm{thr}}\right)
$$

where $c$ is the energy conversion factor, $p_{0}$ and $p_{1}$ are two constants determined experimentally by studying the dependence between threshold positions (in units of ADC channel) and $V_{\mathrm{thr}}$. In order to determine values of $p_{0}$ and $p_{1}$ in Eq. (2.3), we carried out several runs setting $V_{\text {thr }}$ to different values and taking background data. The left panel of Fig. 3 shows an example of the recorded energy spectrum for a pre-selected channel for a typical background data run. As there are only a few hits amplitudes below the sharp cut-off on the left side of the spectrum we can assume that this effect is caused by the hardware threshold. The spectrum area around the cut-off was fitted with a line as shown in the left panel of Fig. 3. The position of the half-maximum of the fitting range counts gives the threshold position. The same fit procedure was repeated for all test runs. The right panel of Fig. 3 shows threshold positions as a function of $V_{\mathrm{thr}}$. Values of $p_{0}$ and $p_{1}$ were obtained from a linear fit to the presented data points.
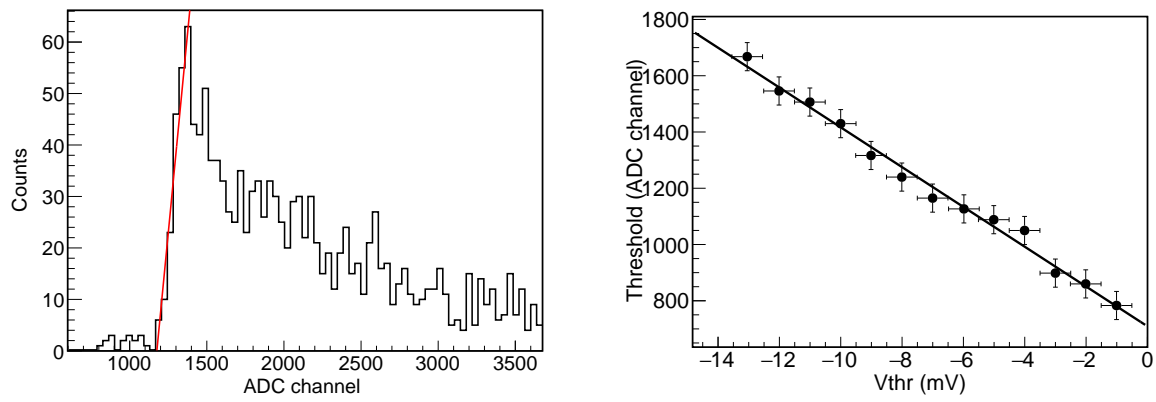

Fig. 3: Example of the threshold position fit (left) and threshold positions as a function of $V_{\text {thr }}$ with a linear fit line (right).

\section{POLAR final setting optimization}

\subsection{Optimization of settings for in-flight calibration}

POLAR has to undergo periodic calibrations during the mission in order to monitor energy response changes in its channels. The instrument gain factors may be susceptible to thermal drift, variations in high voltage values as well as ageing and total dose effects. The in-flight calibration of POLAR is performed with four internal ${ }^{22} \mathrm{Na}$ positron sources using positions of Compton edges in recorded energy spectra. The spectra are extracted with special conditions imposed on coincidence hits produced by x-rays from positron-electron annihilation. The in-flight calibration needs to be performed with several high voltage and threshold settings so that Eq. (2.2) can be properly parametrized with such periodically determined conversion factors. A proper high voltage and threshold setting for the in-flight calibration should meet the following requirements: 1) all 
Compton edges of 1600 channels (at $341 \mathrm{keV}$ ) measured with annihilation gamma-rays are visible, i.e. the minimum detection range is around $400 \mathrm{keV}$ assuming the energy resolution of about 20\%; 2) 25 modules have similar detection efficiencies for the $511 \mathrm{keV}$ gamma-rays emitted by internal calibration sources; 3) Threshold values of different modules are between $20-60 \mathrm{keV}$ and hot channels if any are ignored. By using Eq. (2.2) parametrized from the calibration with the ${ }^{137} \mathrm{Cs}$ source in the laboratory, we calculated the high voltage setting meeting above requirements in all 25 modules. The high voltages are as shown in Fig. 4. The minimum energy range among 64 channels of each module is around $400 \mathrm{keV}$ and the average expected Compton edge positions are at about $60 \%$ of the ADC range. This means that Compton edges should be seen in spectra of all 1600 channels. After knowing the high voltage values the threshold voltage $\left(V_{\text {thr }}\right)$ setting for each module was optimized using Eq. (2.3). The calculated average threshold value for 1600 channels is equal to about $40 \mathrm{keV}$. Due to gain non-uniformities in the modules the real, individual thresholds range from $30 \mathrm{keV}$ to $60 \mathrm{keV}$.

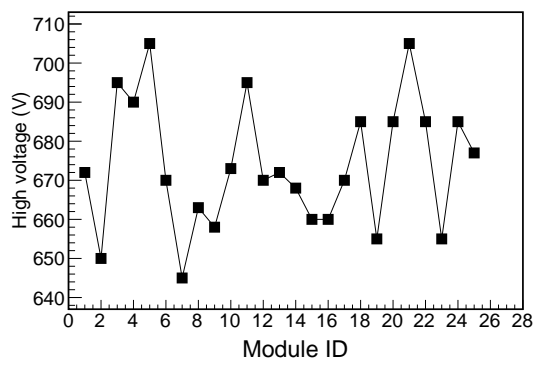

Fig. 4: POLAR basic high voltage setting for in-flight calibration.

In order to validate these calculations, POLAR FM was tested with a $10 \mu \mathrm{Ci}{ }^{137} \mathrm{Cs}$ source in our laboratory. The source was placed on the top of the FM about $15 \mathrm{~cm}$ above its cover. It was moved to the top of different modules during the test. The Compton edges (at $477 \mathrm{keV}$ ) were also expected to be visible at least in energy spectra of the channels with lower gains. Fig. 5 shows an example of experimental and calculated Compton edge positions for 21 channels of POLAR before corrections for temperature effects. The mean temperature in POLAR FM modules during the latest test was equal to $25^{\circ} \mathrm{C}$. It is about 10 degree lower than during previous laboratory calibrations aimed to determine energy conversion factors and high voltage dependence. In addition the temperatures of different modules were in general different. Taking it into account gives finally a good agreement between experimental and calculated positions of Compton edges.

The settings discussed above were stored in the flash memory of the POLAR FM to be used for in-flight calibration runs. In addition, we constructed and optimized another four settings for the same purpose. The high voltage values of each module differed by $-21 \mathrm{~V},-14 \mathrm{~V},-7 \mathrm{~V}$ and $+6 \mathrm{~V}$ relative to the values of the basic calibration setting.

Several in-flight calibration runs of POLAR have already been performed after the launch using all five settings described above with typical results presented below. The first five panels of Fig. 6 show energy spectra for each of the settings constructed using $511 \mathrm{keV}$ coincidence hits for POLAR channel 44. The fit of the Compton edge positions vs. the high voltage values is shown in 


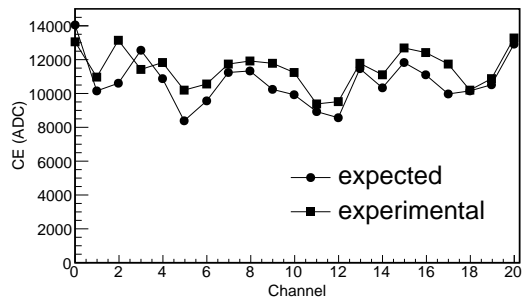

Fig. 5: A Comparison between ${ }^{137} \mathrm{Cs}$ experimental Compton edge positions and calculations.

the sixth panel of Fig. 6. Note that the ADC channel values in the space data packets were scaled down by the factor $1 / 4$ in order to reduce size of the the data packet.
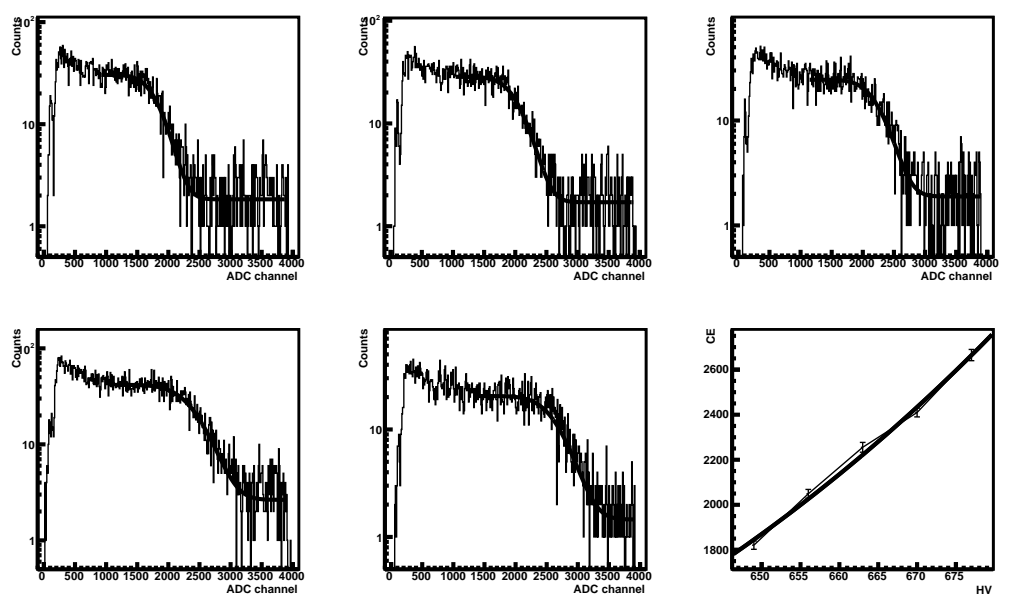

Fig. 6: Example of the POLAR in-flight calibration data for channel 44. Five panels show energy spectra of coincidence hits at five different high voltage values; the last panel plot shows a fit of Compton edge positions vs. high voltage values.

\subsection{Optimization of settings for GRB observation}

Anticipating various signal to background and noise conditions in space we calculated for each module three high voltage and discriminator threshold settings using parametrized Eqs. (2.2) and (2.3). The mean values of thresholds according to calculations were set to energies equal to $15 \mathrm{keV}, 7.5 \mathrm{keV}$ and $5 \mathrm{keV}$. Corresponding high voltage values are shown in Fig. 7. In average all modules were expected to have very close values of energy conversion factors and also the thresholds so that detection efficiencies would be similar. Directly after applying these settings to POLAR FM several hot channels could be observed. Therefore the thresholds in corresponding modules were increased to reduce counting rates and numbers of accidental coincidences caused purely by hot channels. The background counting rates in the laboratory measured for these three final settings were about $220 \mathrm{~Hz}, 400 \mathrm{~Hz}$ and $500 \mathrm{~Hz}$ per module respectively. The measurements were performed in the laboratory with typical temperatures about $20^{\circ} \mathrm{C}$. Further verification in 


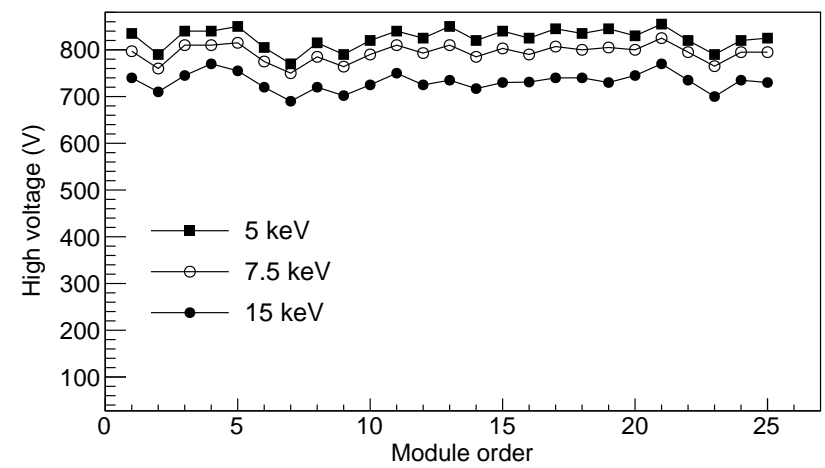

Fig. 7: MAPMT high voltage values of three settings optimized for GRB observation. The mean thresholds of the three settings are $15 \mathrm{keV}, 7.5 \mathrm{keV}$ and $5 \mathrm{keV}$.

space reveal very high levels of the low energy background. In order to reduce the background rate and provide enough resources in the telemetry bandwidth for GRB detections mostly the setting with the mean threshold value of $15 \mathrm{keV}$ have been applied.

\section{Conclusion}

POLAR is a compact wide-field of view, space-borne detector dedicated for precise measurements of the linear polarization of hard X-rays emitted by transient sources in the energy range from $50 \mathrm{keV}$ to $500 \mathrm{keV}$. Based on the parametrized energy conversion factor, their high voltage dependence and the calibrated values of thresholds, sever settings were constructed and optimized for in-flight calibrations and observations of GRBs. In-flight calibrations of POLAR in space were performed successfully for several times. Typical settings for routine operation utilise the one with mean threshold value of $15 \mathrm{keV}$. To date i.e. within six months of operation in space more than 50 GRBs have been already detected.

\section{References}

[1] D. Lazzati, New J. Phys. 8 (8) (2006) 131.

[2] K. Toma, T. Sakamoto, B. Zhang, et al., Astrophys. J. 698 (2009) 1042.

[3] N. Produit ,et al., Nucl. Instr. Meth. Phys. Res. A, 550 (2005) 616 - 625.

[4] S. Orsi,et al., Nucl. Instr. Meth. Phys. Res. A, 648 (1) (2011) 139 - 154.

[5] H. Xiao,W. Hajdas, B. Wu, et al., Astroparticle Physics 83 (2016) 6 - 12.

[6] Hammatstu, Photomultiplier tubes basic and applications, https://www.hamamatsu.com/resources/pdf/etd/PMT_handbook_v3aE.pdf .

[7] H. L. Xiao, W. Hajdas, et al., 2015 IEEE NSS/MIC, 2015,1 - 5. doi:10.1109/NSSMIC.2015.7581896.

[8] X.F. Zhang, W. Hajdas et al., Optimization of the gain factors and parameter settings for the new gamma-ray burst polarimeter, POLAR, submitted to Nucl. Instr. Meth. Phys. Res. A. 